\title{
Asymmetries in Information Processing in a Decision Theory Framework ${ }^{\dagger}$
}

\author{
Luís Santos-Pinto
}

\begin{abstract}
Research in psychology suggests that some individuals are more sensitive to positive than to negative information while others are more sensitive to negative rather than positive information. I take these cognitive positive-negative asymmetries in information processing to a Bayesian decision-theory model and explore its consequences in terms of decisions and payoffs. I show that in monotone decision problems economic agents with more positive-responsive information structures are always better off, ex-ante, when they face problems where payoffs are relatively more sensitive to the action chosen when the state of nature is favorable.
\end{abstract}

JEL Classification Numbers: A12, D81.

Keywords: Information Processing; Decision Theory.

${ }^{\dagger}$ This paper is part of my Ph.D. dissertation in University of California, San Diego. I am indebted to my Ph.D. supervisor, Joel Sobel, for extremely helpful suggestions. I would also like to thank Joel Watson, Vince Crawford, and seminar participants at University of California, San Diego for insightful comments and suggestions. I gratefully acknowledge financial support from Praxis XXI and Fundação Calouste Gulbenkian. 


\section{Introduction}

Information is a key component of most types of economic behavior. When we make our investment decisions, choose what career to follow, decide what type of health insurance coverage we want to have, when to retire, or what is the best schooling district for our kids, we are faced and have to process a wide variety of information and data that may come from published sources, past personal experience, or advice from relatives, friends and experts.

Research in psychology suggests that many individuals are more sensitive to positive than to negative information. According to Matlin and Gawron:

Many individuals (...) recognize pleasant stimuli faster; they judge pleasant stimuli to be more frequent; they use pleasant words more often; they supply a greater number of free associates to pleasant stimuli; they recall pleasant items more accurately, they recall pleasant items earlier in a list; and they process pleasant information more rapidly. (Matlin and Gawron, 1979, pp. 41)

There is also evidence that other individuals are more sensitive to negative than to positive information. According to Lewicka, Czapinski, and Peeters, these individuals have:

(...) stronger cognitive curiosity manifested for negative than for positive stimuli (Fiske, 1980), higher linguistic sophistication for negative than for positive category labels (Clark and Clark, 1977), higher informativeness of negative than of positive personality trait labels (Czapinski, 1986), more rational and normatively appropriate character of inferences applied to negative than to positive targets (Lewicka, 1989). (Lewicka, Czapinski, and Peeters, 1992, pp. 426)

This papers incorporates asymmetries in information processing in a Bayesian decision-theory model and explore its consequences in terms of decisions and payoffs. I assume that while some people are endowed with positive-responsive information processing technologies (from now abbreviated to IPTs) others are endowed with negative-responsive IPTs. This assumption is my main departure from standard decision-theory. Individuals endowed with positive-responsive IPTs have an advantage in processing favorable information while those endowed with negative-responsive IPTs have an advantage in processing unfavorable information.

The model does not assume that individuals suffer from biased information processing or that they are boundedly rational. An information processing technology (IPT) can be thought of as a filter that acts on the sensory system. Just as some individuals have more delicate senses of taste, smell, touch, sight, or sound, than others, individuals endowed with more negative-responsive IPTs are relatively more sensitive to unfavorable information than individuals endowed 
with more positive-responsive IPTs. Thus, individuals endowed with these types of IPTs are not systematically mistaken, they just happen to have different characteristics that determine the way they view the world.

I propose precise definitions of more positive-responsive distribution of beliefs and more positive-responsive information structures. I show that in monotone decision problems agents with more positive-responsive (negative-responsive) information structures are always better off, ex-ante, when they face problems where payoffs are relatively more sensitive to the action chosen when the state of nature is favorable (unfavorable).

Just as a person with a highly sensitive sense of sound has an advantage in music (and may turn out to be a music performer or a composer) or a person with a highly sensitive sense of taste has an advantage in cooking (and may turn out to be a chef), an individual with a positive-responsive (negative-responsive) information structure has an advantage in decision problems where payoffs are relatively more sensitive to the action chosen when the information about the state of nature is favorable (unfavorable).

The paper proceeds as follows. Section 2 sets-up of the decision problem. Section 3 explains how information structures can be compared. Section 4 introduces the positive-responsive order for posterior beliefs. Section 5 introduces the set of incremental return functions associated with the positive-responsive order for posterior beliefs. Section 6 describes the main result and an economic application. Section 7 concludes the paper. Proofs of propositions are in the Appendix.

\section{Set-up}

Consider a Bayesian decision-theory framework where a decision-maker must choose an action or control variable (effort, output, prices) in order to maximize expected utility, which depends of both the control variable and of a random variable, or state of nature, that is not controlled by the decision-maker. Before choosing the control variable the decision-maker obtains information concerning the random variable. This information leads the decision-maker to update her beliefs about the state of nature and therefore make better decisions by comparison with a situation where the decision maker is only in possession of his prior probability assessment of the realization of each state of nature.

The timing of the model is as follows:

1. Nature draws a state realization unobservable by the decision-maker;

2. Nature draws an imperfect signal about the true state realization;

3. The decision-maker processes the signal according to her information processing technology;

4. The decision-maker updates her beliefs about the state of nature and chooses the optimal action; 
5. The payoff to the decision-maker is determined jointly by the action chosen and the state of nature realization.

Thus, for any two decision-makers endowed with different information processing technologies that face the same signaling technology, the one endowed with a "more positive-responsive" information processing technology processes signals in such a way that she ends up with a "more positive-responsive" information structure than the decision-maker endowed with the "less positiveresponsive" information processing technology. That is, decision-makers endowed with positive-responsive IPTs have positive-responsive information structures and decision-makers endowed with negative-responsive IPTs have negativeresponsive information structures. Since, for all purposes, all that matters for individuals to make decisions is their information structures I do not need to model information processing technologies. ${ }^{1}$

The decision problem is composed of the following five elements.

1. A probability space $(\Omega, \mathcal{B}, \mu)$, where $\Omega$ is the set of possible realizations of the state of nature, with $\Omega=[a, b] \subset \Re, \mathcal{B}$ is the Borel $\sigma$-field of $\Omega$ and $\mu$ is a probability measure on $(\Omega, \mathcal{B})$, with $\mu(\Omega)=1$.

2. Two real-valued random variables $X$ and $W$, where $X$ is the signal function with typical realization $x \subset \mathcal{X}$, with $\mathcal{X}=[c, d] \subset \Re$, and $W$ is the state of nature function with typical realization $\omega \subset \Omega$.

3. A joint probability distribution, $F: \Omega \times \mathcal{X} \rightarrow[0,1]$, that will be called the decision-maker's information structure and has generic element $F(W=$ $\omega, X=x)$ or, in condensed notation, $F(\omega, x)$. Note that the two random variables, $X$ and $W$, are fully characterized by $F$ since from $F$ one can obtain (1) the marginal probability distribution of the signal, $F_{X}: \mathcal{X} \rightarrow[0,1]$, with generic element $F_{X}(X=x)$ or, in condensed notation, $F_{X}(x),(2)$ the decision maker's prior beliefs or the marginal probability distribution of the state of nature, $F_{W}: \Omega \rightarrow[0,1]$, with generic element $F_{W}(W=\omega)$, or, in condensed notation $F_{W}(\omega)$, and (3) the decision maker's posterior beliefs after observing a signal realization $X=x$ or the conditional distribution of $W$ given $X=x, F_{W}(\cdot \mid x): \Omega \rightarrow[0,1]$, with generic element $F_{W}(W=\omega \mid X=x)$ or, in condensed notation, $F_{W}(\omega \mid x)$.

4. A set of actions, $\mathcal{A}$, with typical element $a \in \mathcal{A}$.

5. A payoff function, $w(\omega, a), w: \Omega \times \mathcal{A} \rightarrow \Re .^{2}$

\footnotetext{
${ }^{1}$ Alternatively, I could have modelled an information system composed of three elements: (1) a signaling technology (2) an information processing technology, and (3) an information structure. The information processing technology transforms signals into other signals, is unknown to and out of the control of the decision-maker. This approach is less parcimonious as the one I use here.

${ }^{2}$ To provide a better understanding of the payoff function I compare it to the von NeumannMorgenstern utility function. Let $X$ be the set of consequences, outcomes or prizes, and let $x \in X$ be a typical element of $X$. Let $\rho$ denote the outcome function, a mapping from $\Omega \times \mathcal{A}$
} 
Given these five elements I can define a the decision problem as the tuple $D=\left(F, w, F_{W}\right)$. I am now in a position where I can define the interim value of the decision problem $D$ given a signal realization $X=x$ and the ex ante value of a decision problem $D$. The interim value of the decision problem $D$ given a signal realization $X=x$ is given by the expression

$$
\max _{a \in \mathcal{A}} \int_{\Omega} w(\omega, a) d F_{W}(\omega \mid x) .
$$

The ex ante value of the decision problem $D$ is defined as

$$
V(D)=\int_{\mathcal{X}}\left[\max _{a \in \mathcal{A}} \int_{\Omega} w(\omega, a) d F_{W}(\omega \mid x)\right] d F_{X}(x) .
$$

Let $\mathcal{W}$ denote the set of all payoff functions and let $\mathcal{F}_{W}$ denote the set of all prior probability distributions. A family of decision-makers will be defined by a pair $\left(F_{W}, \mathcal{W}^{P}\right)$ where $F_{W} \in \mathcal{F}_{W}$ and $\mathcal{W}^{P}$ stands for a set of payoff functions that have in common a certain property $P$. Furthermore, define the incremental return function as

$$
r(\omega)=w\left(\omega, a^{\prime}\right)-w(\omega, a), \forall a^{\prime}>a .
$$

Most of the analysis that follows will focus on the incremental return function and not on the payoff function directly. However, both functions are intimately related since the payoff of any action for any typical state of nature $\omega$ can be written as a sum of incremental returns. Consider the case where $\mathcal{A}$ is a finite set, $\mathcal{A}=\left\{a_{1}, a_{2}, \ldots, a_{n}\right\}$, then we have

$$
\begin{aligned}
w\left(\omega, a_{k}\right) & =w\left(\omega, a_{1}\right)+\left[w\left(\omega, a_{2}\right)-w\left(\omega, a_{1}\right)\right]+\ldots+\left[w\left(\omega, a_{k}\right)-w\left(\omega, a_{k-1}\right)\right] \\
& =w\left(\omega, a_{1}\right)+\sum_{i=2}^{k} r_{i}(\omega) .
\end{aligned}
$$

Following Athey and Levin (1997) I let

$$
\mathcal{R}=\{g: \Omega \rightarrow \Re, g \text { bounded and measurable }\},
$$

and consider that a payoff function $w$ has an $R_{Q}$ incremental return function if for any $a^{\prime}>a$ we have $r(\omega)=w\left(\omega, a^{\prime}\right)-w(\omega, a) \in R_{Q} \subset \mathcal{R}$.

The set of payoff functions with a $R_{Q}$ incremental return function will be denoted by $\mathcal{W}^{R_{Q}}$. For example, if the incremental return function is nondecreasing, then the set of payoff functions whose incremental return functions are nondecreasing will be denoted by $\mathcal{W}^{R_{N D}}$.

to $X$. The outcome function specifies the consequence, outcome, or prize resulting from each state-action pair, $x=\rho(\omega, a)$. Let $u$ denote the von Neumann-Morgenstern utility function, a mapping from $X$ to $\Re$. The payoff function is equivalent to the successive application of the outcome function and the von Neumann-Morgenstern utility function. That is, $w(\omega, a) \equiv$ $u[\rho(\omega, a)]=u(x)$. Thus, the payoff function is a combined expression of an individual's preferences and of her explanation of the outcome as determined by her action and the state of nature. 


\section{Comparison of Information Structures}

Blackwell $(1951,1953)$ has defined a partial order (we will denote it by $\succsim_{B L}$ ) on the set of all information structures that says that an information structure, $F^{\prime}$, is "more informative" than another, $F$, if and only if, for any payoff function and any prior distribution, the ex ante value of the decision problem when the decision-maker uses $F^{\prime}$ is at least as large as the ex ante value of the decision problem when the decision-maker uses $F$, that is

$$
F^{\prime} \succsim_{B L} F \Leftrightarrow V\left(F^{\prime}, w, F_{W}\right) \geq V\left(F, w, F_{W}\right), \forall w \in \mathcal{W}, \forall F_{W} \in \mathcal{F}_{W} .
$$

This partial ordering of information structures says that more variable beliefs are desirable for any decision-maker, regardless of her attitude towards risk, since greater variability of beliefs means that signal realizations convey more information about the true state of nature.

The problem we face is of a different kind since we wish to find out in what types of economic environments does an individual with a more positiveresponsive information structure outperform an individual with a less positiveresponsive information structure. To answer this question we need to find the appropriate "more positive-responsive" order of information structures (we will denote it by $\left.\succsim_{P R}\right)$ and the right property of the incremental return function (we will denote it by $R_{P R}$ ), such that

$$
F^{\prime} \succsim_{P R} F \Leftrightarrow V\left(F^{\prime}, w, F_{W}\right) \geq V\left(F, w, F_{W}\right), \forall w \in \mathcal{W}^{R_{P R}}, \forall F_{W} \in \mathcal{F}_{W} .
$$

We need a notion of valuable information that is related to concrete economic environments and that necessarily does not hold across all economic environments as Blackwell's notion does. Thus, my "more positive-responsive" order will necessarily have to rank information structures that are not comparable using Blackwell's "more informative" order.

Athey and Levin (1997) present a framework that provides conditions for decision makers with different payoff functions to rank two information structures according to stochastic orders tailored for specific economic environments. Their approach rests in three steps. First, they specify a property of the incremental returns function that induces a stochastic order on posterior beliefs. Second, they restrict their analysis to monotone decision problems. Third, they propose a monotone information order for ranking information structures. My approach only differs from Athey and Levin's approach in the sense that my starting point is the specification of a stochastic order on posterior beliefs that tries to capture the idea of one distribution of posterior beliefs being "more positive-responsive" than another. After having specified the adequate order on posterior beliefs I search for the appropriate property of the incremental returns

function that induces it. Finally, I use Athey and Levin's monotone information order to rank information structures as more or less positive-responsive. 


\section{$4 \quad$ PR Order for Posterior Beliefs}

The PNA theory suggests that decision-makers endowed with negative-responsive IPTs are relatively more sensitive to perceiving unfavorable information than those endowed with positive-responsive IPTs. I need to turn this statement into an operational definition.

One possible interpretation is that a decision-maker endowed with a negativeresponsive IPT is able to distinguish more accurately between different degrees of unfavorable information and is not able to distinguish as accurately between different degrees of favorable information when compared with a decision-maker endowed with a positive-responsive IPT. This interpretation has a direct translation in terms of the dispersion of posterior beliefs: a "more positive-responsive" posterior distribution must have a greater dispersion of beliefs in favorable states of nature and a smaller dispersion of beliefs in unfavorable states than a "less positive-responsive" posterior distribution.

This suggests that an appropriate transfer of dispersion from the right to the left in a "more positive-responsive" posterior distribution will give us a "less positive-responsive" posterior distribution. However, this transfer of dispersion must be balanced. If we reduce the dispersion in the right side by a very small amount and increase the dispersion in the left side by a large amount it is most likely that the obtained (less positive-responsive) posterior distribution is overall more informative (in Blackwell's sense) than the initial (more positiveresponsive) posterior distribution. So, we wish that the transfer of dispersion in a distribution is done in such a way that it does not change its overall dispersion but simply changes the placement of dispersion.

One possible approach that obeys this criteria is to take as measure of overall dispersion of a distribution its variance and restrict attention to comparing distributions that have the same mean and variance and differ only in terms of placement of dispersion. Basically, this approach says that a posterior distribution of beliefs is more positive-responsive than another if it has more upside dispersion of beliefs, or, a posterior distribution of beliefs is more negativeresponsive than another if it has more downside dispersion of beliefs.

This approach parallels the one adopted by Menezes, Geiss, and Tressler (1980) for the characterization of increasing downside risk. Thus, my definition of a "more positive-responsive" distribution of posterior beliefs mirrors Menezes, Geiss, and Tressler's concept of a "more upside risky" distribution of payoffs. ${ }^{3}$

Definition 1 The distribution of posterior beliefs $F_{W}\left(\cdot \mid x^{\prime}\right)$ is more positiveresponsive than $F_{W}(\cdot \mid x)$ if $F_{W}(\cdot \mid x)$ can be obtained from $F_{W}\left(\cdot \mid x^{\prime}\right)$ by a sequence of mean-variance-preserving transformations that shift dispersion of beliefs from the right to the left.

Menezes, Geiss, and Tressler introduced to the economics literature the concept of a mean-variance-preserving transformation (MVPT): a combination of a

\footnotetext{
${ }^{3} \mathrm{~A}$ more negative-responsive (positive-responsive) distribution of posterior beliefs in my setting is the counterpart to a more downside (upside) risky distribution of payoffs in Menezes, Geiss, and Tressler's setting.
} 
mean-preserving spread (MPS) and a mean-preserving contraction (MPC) that, when applied to a distribution, preserves its variance. In a MVPT the MPS is applied everywhere before or after the MPC. ${ }^{4}$

Example: Table I illustrates a single MVPT that transforms $f_{W}\left(\cdot \mid x^{\prime}\right)$, the more positive-responsive distribution, into $f_{W}(\cdot \mid x)$, the less positive-responsive distribution, by shifting dispersion of beliefs from the right to the left.

Table I

\begin{tabular}{|c|c|c|c|c|c|}
\hline & $\omega=0$ & $\omega=1$ & $\omega=2$ & $\omega=3$ & $\omega=4$ \\
\hline $\mathrm{A}$ & $18 / 80$ & $18 / 80$ & $16 / 80$ & $2 / 80$ & $26 / 80$ \\
\hline $\mathrm{B}$ & $22 / 80$ & $10 / 80$ & $20 / 80$ & $2 / 80$ & $26 / 80$ \\
\hline $\mathrm{C}$ & $22 / 80$ & $10 / 80$ & $16 / 80$ & $10 / 80$ & $22 / 80$ \\
\hline
\end{tabular}

Line A shows the probability mass function $f_{W}\left(\cdot \mid x^{\prime}\right)$ Line B is obtained after applying a 3-point MPS to the left of $f_{W}\left(\cdot \mid x^{\prime}\right) .{ }^{5}$ Line $\mathrm{C}$ shows the probability mass function $f_{W}(\cdot \mid x)$. Line $\mathrm{C}$ is obtained after applying a 3 -point MPC to the right of the probability mass function represented in line B. It is easy to check that $E\left[W \mid x^{\prime}\right]=E[W \mid x]=2$ and $V\left[W \mid x^{\prime}\right]=V[W \mid x]=196 / 80$.

Menezes, Geiss and Tressler's Theorem 1 gives us an alternative way of characterizing the more positive-responsive order: one can say that $F_{W}\left(\cdot \mid x^{\prime}\right)$ is more positive-responsive than $F_{W}(\cdot \mid x)$ if and only if

$$
\begin{gathered}
E\left(W \mid x^{\prime}\right)=E(W \mid x) \\
V\left(W \mid x^{\prime}\right)=V(W \mid x) \\
\int_{a}^{\omega} \int_{a}^{t} F_{W}\left(z \mid x^{\prime}\right) d z d t \geq \int_{a}^{\omega} \int_{a}^{t} F_{W}(z \mid x) d z d t, \forall \omega \in[a, b] .
\end{gathered}
$$

\section{$5 \quad \boldsymbol{R}_{P R}$ Incremental Return Functions}

Having specified the more positive-responsive order on posterior beliefs we are now in a position were we can search for the appropriate property of the incremental return function that induces it. The first thing to note is that inequality (5) is related to third-order stochastic dominance. To see this let us recall that the three stochastic dominance orders most commonly used in economics are first, second and third-order stochastic dominance, FOSD, SOSD, and TOSD, respectively. These three orders, in our context, are defined as follows:

FOSD: $F_{W}\left(\cdot \mid x^{\prime}\right) \succsim_{F O S D} F_{W}(\cdot \mid x)$ if $F_{W}\left(\cdot \mid x^{\prime}\right) \neq F_{W}(\cdot \mid x)$

$$
\text { and } F_{W}\left(\cdot \mid x^{\prime}\right) \leq F_{W}(\cdot \mid x), \forall \omega \in[a, b]
$$

\footnotetext{
${ }^{4}$ I will use the term MPS (and MPC) as defined by Landsberger and Meilijson's (1990). Their definition of a MPS avoids the drawbacks of Rothschild and Stiglitz's (1970) definition.

${ }^{5}$ See Rasmusen and Petrakis (1992) for an illustration of the differences between a 4-point MPS, the one used by Rothschild and Stiglitz (1970), and a 3-point MPS.
} 
SOSD: $F_{W}\left(\cdot \mid x^{\prime}\right) \succsim_{S O S D} F_{W}(\cdot \mid x)$ if $F_{W}\left(\omega \mid x^{\prime}\right) \neq F_{W}(\omega \mid x)$

$$
\text { and } \int_{a}^{\omega} F_{W}\left(t \mid x^{\prime}\right) d t \leq \int_{a}^{\omega} F_{W}(t \mid x) d t, \forall \omega \in[a, b]
$$

TOSD: $F_{W}\left(\cdot \mid x^{\prime}\right) \succsim_{T O S D} F_{W}(\cdot \mid x)$ if $F_{W}\left(\omega \mid x^{\prime}\right) \neq F_{W}(\omega \mid x), E\left[W \mid x^{\prime}\right] \geq E[W \mid x]$

$$
\text { and } \int_{a}^{\omega} \int_{a}^{t} F_{W}\left(z \mid x^{\prime}\right) d z d t \leq \int_{a}^{\omega} \int_{a}^{t} F_{W}(z \mid x) d z d t, \forall \omega \in[a, b] .
$$

Thus, given conditions (3), (4), and (5) we see that if $F_{W}\left(\cdot \mid x^{\prime}\right)$.is more positive-responsive than $F_{W}(\cdot \mid x)$, then $F_{W}\left(\cdot \mid x^{\prime}\right)$ is dominated by $F_{W}(\cdot \mid x)$ by TOSD, that is

$$
F_{W}\left(\cdot \mid x^{\prime}\right) \succsim_{P R} F_{W}(\cdot \mid x) \Rightarrow F_{W}(\cdot \mid x) \succsim_{T O S D} F_{W}\left(\cdot \mid x^{\prime}\right) .
$$

However, it is easy to see that the reverse is not true, that is,

$$
F_{W}(\cdot \mid x) \succsim_{T O S D} F_{W}\left(\cdot \mid x^{\prime}\right) \nRightarrow F_{W}\left(\cdot \mid x^{\prime}\right) \succsim_{P R} F_{W}(\cdot \mid x) .
$$

Now, consider the following three classes of incremental return functions:

$R_{1}=\left\{r(\omega) \in R: r_{\omega}(\omega)>0\right\}$

$R_{2}=\left\{r(\omega) \in R_{1}: r_{\omega \omega}(\omega) \leq 0\right\}$

$R_{3}=\left\{r(\omega) \in R_{2}: r_{\omega \omega \omega}(\omega) \geq 0\right\}$.

The theorems that relate these three classes of incremental return functions with first, second, and third-order stochastic dominance can be stated as follows:

$$
\begin{aligned}
& F_{W}\left(\cdot \mid x^{\prime}\right) \succsim_{F O S D} F_{W}(\cdot \mid x) \Leftrightarrow E\left[r(W) \mid x^{\prime}\right] \geq E[r(W) \mid x], \forall r(\omega) \in R_{1} \\
& F_{W}\left(\cdot \mid x^{\prime}\right) \succsim_{S O S D} F_{W}(\cdot \mid x) \Leftrightarrow E\left[r(W) \mid x^{\prime}\right] \geq E[r(W) \mid x], \forall r(\omega) \in R_{2} \\
& F_{W}\left(\cdot \mid x^{\prime}\right) \succsim_{T O S D} F_{W}(\cdot \mid x) \Leftrightarrow E\left[r(W) \mid x^{\prime}\right] \geq E[r(W) \mid x], \forall r(\omega) \in R_{3}
\end{aligned}
$$

I am looking for a result that is similar to the third theorem. ${ }^{6}$ Defining

$$
R_{P R}=\left\{r(\omega) \in R: r_{\omega \omega \omega}(\omega) \leq 0\right\},
$$

I can state the following result.

Proposition 1 The distribution of posterior beliefs $F_{W}\left(\cdot \mid x^{\prime}\right)$ is more positiveresponsive than $F_{W}(\cdot \mid x)$ if and only if $E\left[r(W) \mid x^{\prime}\right] \geq E[r(W) \mid x]$ for all incremental return functions in $R_{P R}$, or

$$
F_{W}\left(\cdot \mid x^{\prime}\right) \succsim_{P R} F_{W}(\cdot \mid x) \Leftrightarrow E\left[r(W) \mid x^{\prime}\right] \geq E[r(W) \mid x], \forall r(\omega) \in R_{P R} .
$$

The proof of this proposition is the proof of Theorem 2 in Menezes, Geiss and Tressler's adapted to this context. To illustrate clearly the role that the

\footnotetext{
${ }^{6}$ See Hadar and Russel (1969) and Whitmore (1970). The third theorem says that if $F_{W}\left(\cdot \mid x^{\prime}\right)$ dominates $F_{W}(\cdot \mid x)$ by TOSD then the expected incremental returns under $F_{W}\left(\cdot \mid x^{\prime}\right)$ are weakly greater than the expected incremental returns under $F_{W}(\cdot \mid x)$ for all incremental return functions increasing, concave and with a convex first derivative.
} 
imposition of equal means and equal variances plays I will show only how the if part is proved, that is how

$$
F_{W}\left(\cdot \mid x^{\prime}\right) \succsim_{P R} F_{W}(\cdot \mid x) \Rightarrow E\left[r(W) \mid x^{\prime}\right] \geq E[r(W) \mid x], \forall r(\omega) \in R_{P R} .
$$

By definition of expectation and using integration by parts one obtains ${ }^{7}$

$$
\begin{aligned}
E\left[r(W) \mid x^{\prime}\right]-E[r(W) \mid x] & =-r_{\omega}(b)\left[E(W \mid x)-E\left(W \mid x^{\prime}\right)\right] \\
& +\frac{1}{2} r_{\omega \omega}(b)\left\{\left[V\left(W \mid x^{\prime}\right)-V(W \mid x)\right]\right. \\
& \left.+\left[E(W \mid x)-E\left(W \mid x^{\prime}\right)\right]\left[2 b-E\left(W \mid x^{\prime}\right)-E(W \mid x)\right]\right\} \\
& -\int_{a}^{b} r_{\omega \omega \omega}(\omega)\left\{\int_{a}^{\omega} \int_{a}^{t}\left[F_{W}\left(z \mid x^{\prime}\right)-F_{W}(z \mid x)\right] d z d t\right\} d \omega
\end{aligned}
$$

Using the fact the more positive-responsive order imposes equal means, $E\left(W \mid x^{\prime}\right)=$ $E(W \mid x)$, and equal variances, $V\left(W \mid x^{\prime}\right)=V(W \mid x)$, (8) simplifies to

$$
\begin{aligned}
& E\left[r(W) \mid x^{\prime}\right]-E[r(W) \mid x] \\
& =-\int_{a}^{b} r_{\omega \omega \omega}(\omega)\left\{\int_{a}^{\omega} \int_{a}^{t}\left[F_{W}\left(z \mid x^{\prime}\right)-F_{W}(z \mid x)\right] d z d t\right\} d \omega .
\end{aligned}
$$

If the posterior distribution $F_{W}\left(\cdot \mid x^{\prime}\right)$ is more positive-responsive than $F_{W}(\cdot \mid x)$ we know by (5) that the term in curly brackets in the right hand side of (9) is always nonnegative. So, for any $r(\omega) \in R_{P R}$ we have that $E\left[r(W) \mid x^{\prime}\right] \geq$ $E[r(W) \mid x]$.

To give some intuition on the economic meaning of the sign of the third derivative of the incremental return function I introduce an example.

Example: Let the payoff function be given by

$$
w(\omega, a)=5^{1.5} a-\frac{a^{2}}{2}(5-\omega)^{1.5}, \text { with } \omega \leq 5 .
$$

Its incremental return function is given by $r(\omega)=w_{a}(\omega, a)=5^{1.5}-a(5-\omega)^{1.5}$. We see that this incremental return function belongs to the set $R_{P R}$ since $r_{\omega \omega \omega}(\omega)=-\frac{3}{8} a(5-\omega)^{-1.5} \leq 0$.

Let $\Omega=\{0,1,2,3,4\}$ and $\mathcal{A}=\left\{1,(5 / 4)^{1.5},(5 / 3)^{1.5},(5 / 2)^{1.5}, 5^{1.5}\right\}$. In table II I represent the payoffs associated with these actions and states

\section{Table II}

\footnotetext{
${ }^{7}$ The proof of equation 7 is relegated to the Appendix.
} 


\begin{tabular}{|c|c|c|c|c|c|}
\hline & $\omega=0$ & $\omega=1$ & $\omega=2$ & $\omega=3$ & $\omega=4$ \\
\hline$a=1$ & 5.6 & 7.1 & 8.6 & 9.8 & 10.7 \\
\hline$a=(5 / 4)^{1.5}$ & 4.7 & 7.8 & 10.6 & 12.9 & 14.6 \\
\hline$a=(5 / 3)^{1.5}$ & -1.8 & 5.5 & 12.0 & 17.5 & 21.7 \\
\hline$a=(5 / 2)^{1.5}$ & -43.2 & -18.3 & 3.6 & 22.1 & 36.4 \\
\hline$a=5^{1.5}$ & -573.8 & -375.0 & -119.8 & -51.8 & 62.5 \\
\hline
\end{tabular}

In table III I represent the incremental returns of the form

$$
r\left(\omega ; a^{*}(\omega), a\right)=w\left(\omega, a^{*}(\omega)\right)-w(\omega, a), \text { where } a^{*}(\omega)=\max _{a \in \mathcal{A}} w(\omega, a) .
$$

Table III

\begin{tabular}{|c|c|c|c|c|}
\hline$\omega=0$ & $\omega=1$ & $\omega=2$ & $\omega=3$ & $\omega=4$ \\
\hline 0 & 0.7 & 2 & 12.3 & 51.8 \\
\hline 0.9 & 0 & 1.4 & 9.2 & 47.9 \\
\hline 7.4 & 2.3 & 0 & 4.6 & 40.8 \\
\hline 48.8 & 26.1 & 8.4 & 0 & 26.1 \\
\hline 579.4 & 382.8 & 131.8 & 73.9 & 0 \\
\hline
\end{tabular}

By looking at the pattern of incremental returns in table III we see that if a decision-maker faces this problem and has noisy information about the state of nature it is worse not taking the no-noise optimal action when the state of nature is favorable ( $\omega$ high) than when the state of nature is unfavorable ( $\omega$ low). For example, when $\omega=0$ the incremental return of taking action $a=1$ instead of taking action $a=(5 / 4)^{1.5}$ is only 0.9 while when $\omega=1$ the incremental return of taking action $a=(5 / 4)^{1.5}$ instead of taking action $a=(5 / 3)^{1.5}$ is 2.3. As the state of nature becomes more favorable the incremental returns of taking the no-noise optimal actions increase. So, in this problem it is advantageous to have more accurate posteriors for high realizations of $\omega$ rather than for low realizations of $\omega$, that is, a decision-maker faced with this problem will be better off with a more positive-responsive information structure.

The previous example shows that the set of payoff functions with incremental return functions with nonpositive third derivative can be interpreted as the set of payoff functions whose payoffs are relatively more sensitive to the action chosen when the state of nature is favorable, in other words, payoff functions where the cost of not taking the no-noise optimal actions is higher in favorable states than in unfavorable states.

\section{PR Order for Information Structures}

We have already defined the more positive-responsive order for posterior beliefs and we know the set of incremental return functions under which a decisionmaker with a more positive-responsive posterior is better off than a decisionmaker with a more negative-responsive posterior. However, we still have to define a more positive-responsive order for information structures. For this purpose I introduce the following definition. 
Definition 2 An information structure $F$ is positive-responsive ordered if $\succsim_{P R}$ is a complete order on $\left\{F_{W}(\cdot \mid x)\right\}_{x \in \mathcal{X}}$, that is, for any $x^{\prime}>x, F_{W}\left(\cdot \mid x^{\prime}\right) \succsim_{P R}$ $F_{W}(\cdot \mid x)$.

Example: The posterior matrix shown in table IV induces a positive-responsive ordered information structure.

Table IV

\begin{tabular}{|c|c|c|l|c|c|}
\hline & $x=.2$ & $x=.4$ & $x=.6$ & $x=.8$ & $x=1$ \\
\hline$\omega=0$ & $27 / 80$ & $26 / 80$ & $22 / 80$ & $18 / 80$ & $17 / 80$ \\
\hline$\omega=1$ & $0 / 80$ & $2 / 80$ & $10 / 80$ & $18 / 80$ & $20 / 80$ \\
\hline$\omega=2$ & $16 / 80$ & $16 / 80$ & $16 / 80$ & $16 / 80$ & $16 / 80$ \\
\hline$\omega=3$ & $20 / 80$ & $18 / 80$ & $10 / 80$ & $2 / 80$ & $0 / 80$ \\
\hline$\omega=4$ & $17 / 80$ & $18 / 80$ & $22 / 80$ & $26 / 80$ & $27 / 80$ \\
\hline
\end{tabular}

I restrict my attention to information structures that are positive-responsive ordered. By imposing this somewhat strong requirement the decision problem becomes monotone. This result is state formally in the next proposition.

Proposition 2 If $w \subset \mathcal{W}^{R_{P R}}$ and an information structure $F$ is positiveresponsive ordered then there exists a decision rule $\alpha(x)$ that is nondecreasing in $x$.

This proposition tells us that if a decision-maker with a payoff function $w \subset \mathcal{W}^{R_{P R}}$ has a positive-responsive ordered information structure she has an optimal decision rule that is monotone, that is, a decision rule where higher signals (more positive-responsive posteriors) lead to higher actions. The additional structure of monotone decision problems enables us to derive necessary and sufficient conditions for decision-makers in certain economic environments to prefer more or less positive-responsive information structures. ${ }^{8}$

Before stating the main result of the paper I just have to define what a more positive-responsive information structure is.

Definition 3 Information structure $F^{\prime}$ is more positive-responsive than information structure $F, F^{\prime} \succsim_{P R} F$, if (i) $F^{\prime}$ and $F$ come from the same prior, (ii) $F^{\prime}$ and $F$ are both positive-responsive ordered and (iii) for $X^{\prime}$ and $X$ being the two signals associated with information structures $F^{\prime}$ and $F$, respectively, we have

$$
F_{W}^{\prime}\left(\cdot \mid X^{\prime} \geq x\right) \succsim_{P R} F_{W}(\cdot \mid X \geq x), \quad \forall x \in[0,1] .
$$

Condition (10), the monotone information order in Athey and Levin's (1997), tells us that information structure $F^{\prime}$ is more positive-responsive than information structure $F$ if, on average, the more positive-responsive posterior beliefs (the ones induced by higher signal realizations) of information structure $F^{\prime}$ are

\footnotetext{
${ }^{8}$ Examples of monotone decision problems arise in many economic relevant contexts, such as production under uncertainty, financial and capital investments, auctions, contracting, adverse selection, and search. See Athey and Levin (1997) for more examples.
} 
more dispersed than the more positive-responsive posterior beliefs of information structure $F$. I am now ready to state my main finding.

Proposition 3 Information structure $F^{\prime}$ is more positive-responsive than information structure $F$ if and only if, for any payoff function in $\mathcal{W}^{R_{P R}}$ and any prior distribution, the ex ante value of the decision problem when the decisionmaker uses $F^{\prime}$ is at least as large as the ex ante value of the decision problem when the decision-maker uses $F$, or

$$
F^{\prime} \succsim_{P R} F \Leftrightarrow V\left(F^{\prime}, w, F_{W}\right) \geq V\left(F, w, F_{W}\right), \forall w \in \mathcal{W}^{R_{P R}}, \forall F_{W} \in \mathcal{F}_{W} .
$$

This proposition tells us that decision-makers with more positive-responsive information structures are always better off, ex-ante, when they face problems where payoffs are relatively more sensitive to the action chosen when the state of nature is favorable. Similarly, it tells us that decision-makers with more negative-responsive information structures are always better off, ex-ante, when they face problems where payoffs are relatively more sensitive to the action chosen when the state of nature is unfavorable.

To illustrate Proposition 3, consider a monopolist who faces cost uncertainty and that has a payoff function given by $w(\omega, q)=P(q) q-C(\omega, q)$. The ex ante value of the monopolist's decision problem is given by

$$
V(D)=\int_{\mathcal{X}}\left[\max _{q \geq 0} \int_{\Omega}[P(q) q-C(\omega, q)] d F_{W}(\omega \mid x)\right] d F_{X}(x) .
$$

Differentiating the payoff function with respect to output we obtain the incremental return function $r(\omega)=M R(q)-M C(\omega, q)$. Differentiating the incremental return function with respect to the state of nature three times we get $r_{\omega \omega \omega}(\omega)=-M C_{\omega \omega \omega}(\omega, q)$. Proposition 3 tells us that if $M C_{\omega \omega \omega}(\omega, q) \geq 0$, then a monopolist with a more positive-responsive information structure is always better off ex ante than if he has a more negative-responsive information structure.

\section{Conclusion}

This papers assumes that positive-negative asymmetries in information processing come from "cold" cognitive differences among people and not from "warm" biases in information processing (e.g., some individuals recall positive information better than negative information whereas others have the same rate of recall for both types of information). The paper finds that individuals who are better at processing positive (negative) information have an absolute advantage in decision problems where payoffs are relatively more sensitive to the action chosen when the state of nature is favorable (unfavorable). 


\section{References}

Athey, S. and Levin J. (2001). "The Value of Information in Monotone Decision Problems," MIT Working Paper 98-24.

Blackwell, D. (1951). "Comparison of Experiments," Proceedings of the Second Berkeley Symposium on Mathematical Statistics, 93-102.

Blackwell, D. (1953). "Equivalent Comparison of Experiments," Annals of Mathematical Statistics, 24, 265-272.

Blackwell, D. and Girshick, M. (1954). Theory of Games and Statistical Decisions, Dover.

Borch, K. (1968). The Economics of Uncertainty, Princeton University Press.

Fiedler, K., Fladung, U., and U. Hemmeter (1987). "A Positivity Bias in Person Memory," European Journal of Social Psychology, Vol. 17, 243-246.

Hadar, J. and Russel W. (1969). "Rules for Ordering Uncertain Prospects," American Economic Review, 59, 25-34.

Hirshleifer, J. and Riley, J. (1992). The Analytics of Uncertainty and Information, Cambridge University Press.

Laffont, J. (1989). The Economics of Uncertainty and Information, MIT Press.

Landsberger, M. and Meilijson, I. (1990). "A Tale of Two Tails: An Alternative Characterization of Comparative Risk," Journal of Risk and Uncertainty, 3, 65-82.

Lewicka, M., Czapinski, J., and G. Peeters (1992). "Positive-Negative Asymmetry or 'When the Heart Needs a Reason,"' European Journal of Social Psychology, Vol. 22, 425-434.

Marschak, J. (1963). "The Payoff-Relevant Description of States and Acts," Econometrica, Vol. 31, No. 4, 719-725.

Marschak, J. and Radner, R. (1972). Economic Theory of Teams, New Haven and London, Yale University Press.

Matlin, M. and Gawron, V. (1979). "Individual Differences in Pollyannaism," Journal of Personality Assessment, 43, 411-412.

Menezes, C., Geiss, C., and Tressler (1980). "Increasing Downside Risk," American Economic Review, 70, No. 5, 921-932.

Milgrom, P. and C. Shannon (1994). "Monotone Comparative Statics," Econometrica, Vol. 62, No. 1, 157-180.

Ormiston, M. (1992). "Deterministic Transformations: Some Comparative Statistics Results," Decision Making under Risk and Uncertainty: New Models and Empirical Findings, Geweke, J. Ed, 43-51.

Rasmusen, E. and Petrakis, E. (1992). "Defining The Mean-Preserving Spread: 3-PT Versus 4-PT," Decision Making under Risk and Uncertainty: New Models and Empirical Findings, Geweke, J. Ed., 53-58.

Rostchild, M. and Stiglitz, J. (1970). "Increasing Risk: I. A Definition," Journal of Economic Theoy, 2, 225-243. 
Scholz, R. (1987). Cognitive Strategies in Stochastic Thinking, Theory and Decision Library, D. Reidel Publishing Company.

Shaked, M. and Shanthikumar, G. (1994). Stochastic Orders and their Applications, Academic Press.

Shannon, C. (1995). "Weak and Strong Monotone Comparative Statics," Economic Theory, 5(2), 209-227.

Whitmore, G. (1970). "Third Degree Stochastic Dominance," American Economic Review, 60, 457-459.

Whitmore, G. and Findlay, M. (1978). Stochastic Dominance, Lexington Books. 


\section{Appendix}

Proof of Equation (8): By definition of expectation we know that

$$
\begin{aligned}
E\left[r(W) \mid x^{\prime}\right]-E[r(W) \mid x] & =\int_{a}^{b} r(\omega)\left[d F_{W}\left(\omega \mid x^{\prime}\right)-d F_{W}(\omega \mid x)\right] \\
& =\int_{a}^{b} r(\omega)\left[f_{W}\left(\omega \mid x^{\prime}\right)-f_{W}(\omega \mid x)\right] d \omega
\end{aligned}
$$

Integrating by parts we obtain

$$
\begin{aligned}
E\left[r(W) \mid x^{\prime}\right]-E[r(W) \mid x]= & \left|r(\omega)\left[F_{W}\left(\omega \mid x^{\prime}\right)-F_{W}(\omega \mid x)\right]\right|_{a}^{b} \\
& -\int_{a}^{b} r_{\omega}(\omega)\left[F_{W}\left(\omega \mid x^{\prime}\right)-F_{W}(\omega \mid x)\right] d \omega .
\end{aligned}
$$

Since $F_{W}\left(a \mid x^{\prime}\right)-F_{W}(a \mid x)=0$ and $F_{W}\left(b \mid x^{\prime}\right)-F_{W}(b \mid x)=0$ we have

$$
E\left[r(W) \mid x^{\prime}\right]-E[r(W) \mid x]=-\int_{a}^{b} r_{\omega}(\omega)\left[F_{W}\left(\omega \mid x^{\prime}\right)-F_{W}(\omega \mid x)\right] d \omega .
$$

Integrating by parts once again we have

$$
\begin{aligned}
E\left[r(W) \mid x^{\prime}\right]-E[r(W) \mid x]= & -\left|r_{\omega}(\omega) \int_{a}^{\omega}\left[F_{W}\left(t \mid x^{\prime}\right)-F_{W}(t \mid x)\right] d t\right|_{a}^{b} \\
& +\int_{a}^{b} r_{\omega \omega}(\omega)\left(\int_{a}^{\omega}\left[F_{W}\left(t \mid x^{\prime}\right)-F_{W}(t \mid x)\right] d t\right) d \omega .
\end{aligned}
$$

Integrating by parts the second term on the left hand side of the above equation we have

$$
\begin{aligned}
E\left[r(W) \mid x^{\prime}\right]-E[r(W) \mid x]=-\left|r_{\omega}(\omega) \int_{a}^{\omega}\left[F_{W}\left(t \mid x^{\prime}\right)-F_{W}(t \mid x)\right] d t\right|_{a}^{b} \\
+\left|r_{\omega \omega}(\omega) \int_{a}^{\omega}\left(\int_{a}^{t}\left[F_{W}\left(z \mid x^{\prime}\right)-F_{W}(z \mid x)\right] d z\right) d t\right|_{a}^{b} \\
-\int_{a}^{b} r_{\omega \omega \omega}(\omega)\left(\int_{a}^{\omega} \int_{a}^{t}\left[F_{W}\left(z \mid x^{\prime}\right)-F_{W}(z \mid x)\right] d z d t\right) d \omega .
\end{aligned}
$$


The above expression can be simplified to

$$
\begin{aligned}
E\left[r(W) \mid x^{\prime}\right]-E[r(W) \mid x] & =-r_{\omega}(b) \int_{a}^{b}\left[F_{W}\left(\omega \mid x^{\prime}\right)-F_{W}(\omega \mid x)\right] d \omega \\
& +r_{\omega \omega}(b) \int_{a}^{b}\left(\int_{a}^{\omega}\left[F_{W}\left(t \mid x^{\prime}\right)-F_{W}(t \mid x)\right] d t\right) d \omega \\
& -\int_{a}^{b} r_{\omega \omega \omega}(\omega)\left(\int_{a}^{\omega} \int_{a}^{t}\left[F_{W}\left(z \mid x^{\prime}\right)-F_{W}(z \mid x)\right] d z d t\right) d \omega .
\end{aligned}
$$

note that

$$
\int_{a}^{b}\left[1-F_{W}\left(\omega \mid x^{\prime}\right)\right] d \omega=-a+E\left(W \mid x^{\prime}\right)
$$

and

$$
\int_{a}^{b}\left[1-F_{W}(\omega \mid x)\right] d \omega=-a+E(W \mid x)
$$

imply that

$$
\int_{a}^{b}\left[F_{W}\left(\omega \mid x^{\prime}\right)-F_{W}(\omega \mid x)\right] d \omega=E(W \mid x)-E\left(W \mid x^{\prime}\right) .
$$

Also,

$$
\int_{a}^{b}\left(\int_{a}^{\omega} F_{W}\left(t \mid x^{\prime}\right) d t\right) d \omega=\frac{b^{2}}{2}-b E\left(W \mid x^{\prime}\right)+\frac{1}{2} E\left(W^{2} \mid x^{\prime}\right),
$$

and

$$
\int_{a}^{b}\left(\int_{a}^{\omega} F_{W}(t \mid x) d t\right) d \omega=\frac{b^{2}}{2}-b E(W \mid x)+\frac{1}{2} E\left(W^{2} \mid x\right),
$$

imply that

$$
\begin{aligned}
\int_{a}^{b}\left(\int _ { a } ^ { \omega } \left[F_{W}\left(t \mid x^{\prime}\right)\right.\right. & \left.\left.-F_{W}(t \mid x)\right] d t\right) d \omega \\
& =-b\left[E\left(W \mid x^{\prime}\right)-E(W \mid x)\right]+\frac{1}{2}\left[E\left(W^{2} \mid x^{\prime}\right)-E\left(W^{2} \mid x\right)\right] .
\end{aligned}
$$


Using the definition of variance we can write the above equation as

$$
\begin{aligned}
& \int_{a}^{b}\left(\int_{a}^{\omega}\left[F_{W}\left(t \mid x^{\prime}\right)-F_{W}(t \mid x)\right] d t\right) d \omega \\
& =\frac{1}{2}\left\{\left[V\left(W \mid x^{\prime}\right)-V(W \mid x)\right]\right. \\
& \left.+\left[E(W \mid x)-E\left(W \mid x^{\prime}\right)\right]\left[2 b-E\left(W \mid x^{\prime}\right)-E(W \mid x)\right]\right\} .
\end{aligned}
$$

Substituting (12) and (13) into (11) we obtain (8).

Q.E.D.

Proof of Proposition 2: This proof is a particular case of the proof of Lemma 1 in Athey and Levin's (1997). Define $U(x, a)=\int_{\Omega} w(\omega, a) d F_{W}(\omega \mid x)$ and let the optimal action for signal realization $X=x$ be defined as $\alpha(x)=$ $\arg \max _{a \in \mathcal{A}} U(x, a)$. We wish to show that if $w \subset \mathcal{W}^{R_{P R}}$ and an information structure $F$ is positive-responsive ordered then there exists an optimal decision rule, $\alpha(\cdot)$, that is nondecreasing in $x$. We know from Shannon's (1995) that if $\mathcal{X}$ is a partially ordered set and $U: \mathcal{X} \times \mathcal{A} \rightarrow \Re$, where $\mathcal{A} \subset \Re$, we have that $\arg \max _{a \in \mathcal{A}} U(x, a)$ is nondecreasing in $x$ if and only if $U(x, a)$ satisfies the single crossing property in $a$. Thus, since $\mathcal{X} \subset \Re$, to prove this proposition we just need to show that $U(x, a)$ satisfies the single crossing property in $a$.

Let $a^{\prime}>a$. We know that since $w \subset \mathcal{W}^{R_{P R}}$ then $r(\omega)=w\left(\omega, a^{\prime}\right)-w(\omega, a) \in$ $R_{P R}$. Given that $F$ is positive-responsive ordered we also have that $\forall x^{\prime}>x$, $F_{W}\left(\cdot \mid x^{\prime}\right) \succsim_{P R} F_{W}(\cdot \mid x)$. By Proposition $1, F_{W}\left(\cdot \mid x^{\prime}\right) \succsim_{P R} F_{W}(\cdot \mid x)$ implies

$$
\begin{aligned}
E\left[r(W) \mid x^{\prime}\right] & \geq E[r(W) \mid x] \\
\int_{\Omega} r(\omega) d F_{W}\left(\omega \mid x^{\prime}\right) d \omega & \geq \int_{\Omega} r(\omega) d F_{W}(\omega \mid x) d \omega \\
\int_{\Omega}\left[w\left(\omega, a^{\prime}\right)-w(\omega, a)\right] d F_{W}\left(\omega \mid x^{\prime}\right) & \geq \int_{\Omega}\left[w\left(\omega, a^{\prime}\right)-w(\omega, a)\right] d F_{W}(\omega \mid x) \\
U\left(x^{\prime}, a^{\prime}\right)-U\left(x^{\prime}, a\right) & \geq U\left(x, a^{\prime}\right)-U(x, a)
\end{aligned}
$$

The last inequality implies that $\forall a^{\prime}>a, U\left(x, a^{\prime}\right)-U(x, a) \geq 0 \Rightarrow U\left(x^{\prime}, a^{\prime}\right)-$ $U\left(x^{\prime}, a\right) \geq 0, \forall x^{\prime}>x$, that is, $U(x, a)$ satisfies the single crossing property in $a$.

Q.E.D.

Proof of Proposition 3: This proof is a particular case of the proof of Theorem 3 in Athey and Levin's (1997). Suppose $\mathcal{A}=\left\{a_{1}, \ldots, a_{i}, \ldots, a_{n}\right\}$ and $\Omega=\left\{\omega_{1}, \ldots, \omega_{j}, \ldots, \omega_{J}\right\}$, that is the action space and the space of state of nature realizations is finite. ${ }^{9}$ We need to show that for any optimal monotone decision rule based on observing signal $X, \alpha(\cdot)$, there is an alternative optimal monotone decision rule based on observing signal $X^{\prime}, \alpha^{\prime}(\cdot)$, that makes a decision-maker ex-ante at least as well.

\footnotetext{
${ }^{9}$ The proof holds if either $\mathcal{A}, \Omega$, or both, are compact sets. See Athey and Levin (1997).
} 
By Lemma 2 in Athey and Levin we know that (10) holds if and only if for all $x \in[0,1]$ and $r \in R_{P R}$

$$
\int_{\Omega} r(\omega) d F_{W}^{\prime}\left(\omega \mid X^{\prime} \geq x\right) \operatorname{Pr}\left(X^{\prime} \geq x\right) \geq \int_{\Omega} r(\omega) d F_{W}(\omega \mid X \geq x) \operatorname{Pr}(X \geq x),
$$

or

$$
E_{W}\left[r(\omega) \mid X^{\prime} \geq x\right] \operatorname{Pr}\left(X^{\prime} \geq x\right) \geq E_{W}[r(\omega) \mid X \geq x] \operatorname{Pr}(X \geq x) .
$$

Let the optimal monotone decision rule associated with information structure $F$ be defined by a set of cut points: $x_{1} \leq x_{2} \leq \ldots \leq x_{n+1}$, with $\alpha(x)=a_{i}$ when $x_{i}<x<x_{i+1}$. The ex-ante expected payoff of using $\alpha(\cdot)$ with information structure $F$ is given by

$$
\begin{aligned}
V\left(F, w, F_{W}, \alpha\right) & =\sum_{j=1}^{m} \sum_{i=1}^{n} w\left(\omega_{j}, \alpha\left(x_{i}\right)\right) f\left(\omega_{j}, x_{i}\right) \\
& =\sum_{j=1}^{m} \sum_{i=1}^{n} w\left(\omega_{j}, a_{i}\right) f\left(\omega_{j}, x_{i}\right) \\
& =\sum_{j=1}^{m}\left[w\left(\omega_{j}, a_{1}\right) f\left(\omega_{j}, x_{1}\right)+\ldots+w\left(\omega_{j}, a_{n}\right) f\left(\omega_{j}, x_{n}\right)\right] .
\end{aligned}
$$

Making use of (2) we have that

$$
\begin{aligned}
V\left(F, w, F_{W}, \alpha\right) & =\sum_{j=1}^{m}\left\{w\left(\omega_{j}, a_{1}\right) f\left(\omega_{j}, x_{1}\right)+\left[w\left(\omega_{j}, a_{1}\right)+r_{2}\left(\omega_{j}\right)\right] f\left(\omega_{j}, x_{2}\right)\right. \\
& +\left[w\left(\omega_{j}, a_{1}\right)+r_{2}\left(\omega_{j}\right)+r_{3}\left(\omega_{j}\right)\right] f\left(\omega_{j}, x_{3}\right)+\ldots \\
& \left.+\left[w\left(\omega_{j}, a_{1}\right)+r_{2}\left(\omega_{j}\right)+\ldots+r_{n}\left(\omega_{j}\right)\right] f\left(\omega_{j}, x_{n}\right)\right\}
\end{aligned}
$$

Collecting terms (15) becomes

$$
\begin{aligned}
& V\left(F, w, F_{W}, \alpha\right)=\sum_{j=1}^{m}\left\{w\left(\omega_{j}, a_{1}\right)\left[f\left(\omega_{j}, x_{1}\right)+f\left(\omega_{j}, x_{2}\right)+\ldots+f\left(\omega_{j}, x_{n}\right)\right]\right. \\
& +r_{2}\left(\omega_{j}\right)\left[f\left(\omega_{j}, x_{2}\right)+f\left(\omega_{j}, x_{3}\right)+\ldots+f\left(\omega_{j}, x_{n}\right)\right] \\
& +r_{3}\left(\omega_{j}\right)\left[f\left(\omega_{j}, x_{3}\right)+\ldots+f\left(\omega_{j}, x_{n}\right)\right]+\ldots+r_{n}\left(\omega_{j}\right) f\left(\omega_{j}, x_{n}\right)
\end{aligned}
$$

or

$$
\begin{aligned}
V\left(F, w, F_{W}, \alpha\right)=\sum_{j=1}^{m}\{w & \left(\omega_{j}, a_{1}\right) \sum_{i=1}^{n} f\left(\omega_{j}, x_{i}\right)+r_{2}\left(\omega_{j}\right) \sum_{i=2}^{n} f\left(\omega_{j}, x_{i}\right) \\
& \left.+r_{3}\left(\omega_{j}\right) \sum_{i=3}^{n} f\left(\omega_{j}, x_{i}\right)+\ldots+r_{n}\left(\omega_{j}\right) f\left(\omega_{j}, x_{n}\right)\right\}
\end{aligned}
$$


or

$$
\begin{gathered}
V\left(F, w, F_{W}, \alpha\right)=\sum_{j=1}^{m}\left\{w\left(\omega_{j}, a_{1}\right) f\left(\omega_{j}\right)+r_{2}\left(\omega_{j}\right) \frac{\sum_{i=2}^{n} f\left(\omega_{j}, x_{i}\right)}{\operatorname{Pr}\left(X \geq x_{2}\right)} \operatorname{Pr}\left(X \geq x_{2}\right)\right. \\
\left.+r_{3}\left(\omega_{j}\right) \frac{\sum_{i=3}^{n} f\left(\omega_{j}, x_{i}\right)}{\operatorname{Pr}\left(X \geq x_{3}\right)} \operatorname{Pr}\left(X \geq x_{3}\right)+\ldots+r_{n}\left(\omega_{j}\right) \frac{f\left(\omega_{j}, x_{n}\right)}{\operatorname{Pr}\left(X \geq x_{n}\right)} \operatorname{Pr}\left(X \geq x_{n}\right)\right\}
\end{gathered}
$$

or

$$
\begin{gathered}
V\left(F, u, F_{W}, \alpha\right)=\sum_{j=1}^{m} u\left(\omega_{j}, a_{1}\right) f\left(\omega_{j}\right)+\sum_{j=1}^{m} r_{2}\left(\omega_{j}\right) \frac{\sum_{i=2}^{n} f\left(\omega_{j}, x_{i}\right)}{\operatorname{Pr}\left(X \geq x_{2}\right)} \operatorname{Pr}\left(X \geq x_{2}\right) \\
+\sum_{j=1}^{m} r_{3}\left(\omega_{j}\right) \frac{\sum_{i=3}^{n} f\left(\omega_{j}, x_{i}\right)}{\operatorname{Pr}\left(X \geq x_{3}\right)} \operatorname{Pr}\left(X \geq x_{3}\right)+\ldots+\sum_{j=1}^{m} r_{n}\left(\omega_{j}\right) \frac{f\left(\omega_{j}, x_{n}\right)}{\operatorname{Pr}\left(X \geq x_{n}\right)} \operatorname{Pr}\left(X \geq x_{n}\right)
\end{gathered}
$$

or

$$
\begin{aligned}
& V\left(F, w, F_{W}, \alpha\right)=E\left[w\left(W, a_{1}\right)\right]+E\left[r_{2}(W) \mid X \geq x_{2}\right] \operatorname{Pr}\left(X \geq x_{2}\right) \\
& +E\left[r_{3}(W) \mid X \geq x_{3}\right] \operatorname{Pr}\left(X \geq x_{3}\right)+\ldots+E\left[r_{n}(W) \mid X \geq x_{n}\right] \operatorname{Pr}\left(X \geq x_{n}\right)
\end{aligned}
$$

or, finally,

$$
V\left(F, w, F_{W}, \alpha\right)=E\left[w\left(W, a_{1}\right)\right]+\sum_{i=2}^{n} E\left[r_{i}(W) \mid X \geq x_{i}\right] \operatorname{Pr}\left(X \geq x_{i}\right) .
$$

Let the optimal monotone decision rule associated with information structure $F^{\prime}$ be defined by the set of cut points: $x_{1}^{\prime} \leq x_{2}^{\prime} \leq \ldots \leq x_{n+1}^{\prime}$, with $\alpha^{\prime}(x)=a_{i}$ when $x_{i}^{\prime}<x<x_{i+1}^{\prime}$. The ex-ante expected payoff of using $\alpha^{\prime}(\cdot)$ with information structure $F^{\prime}$ is given by

$$
V\left(F^{\prime}, w, F_{W}, \alpha^{\prime}\right)=E\left[w\left(W, a_{1}\right)\right]+\sum_{i=2}^{n} E\left[r_{i}(W) \mid X^{\prime} \geq x_{i}\right] \operatorname{Pr}\left(X^{\prime} \geq x_{i}\right) .
$$

Subtracting (16) from (17) we obtain

$$
\begin{aligned}
V\left(F^{\prime}, w, F_{W}, \alpha^{\prime}\right)-V\left(F, w, F_{W}, \alpha\right)= & \sum_{i=2}^{n} E\left[r_{i}(W) \mid X^{\prime} \geq x_{i}\right] \operatorname{Pr}\left(X^{\prime} \geq x_{i}\right) \\
& -\sum_{i=2}^{n} E\left[r_{i}(W) \mid X \geq x_{i}\right] \operatorname{Pr}\left(X \geq x_{i}\right)
\end{aligned}
$$

We see that (14) implies $V\left(F^{\prime}, w, F_{W}, \alpha^{\prime}\right) \geq V\left(F, w, F_{W}, \alpha\right)$ Q.E.D. 